\title{
ESTUDO DO EFEITO DAS COORDENADAS TERMODINÂMICAS SOBRE O ESCOAMENTO NO INTERIOR DE UMA CÂMARA DE PRECIPITAÇÃO DE PARTÍCULAS
}

\author{
R. A. ALMEIDA ${ }^{1}$, R. V. P. REZENDE ${ }^{2}$, H.F. MEIER ${ }^{3}$ D. NORILER ${ }^{3}$, V.F. CABRAL ${ }^{1}$, L. \\ CARDOZO-FILHO ${ }^{1}$ e F. A. R. CARDOSO ${ }^{4}$. \\ ${ }^{1}$ Universidade Estadual de Maringá, Departamento de Engenharia Química \\ ${ }^{2}$ Universidade Federal de Santa Catarina, Departamento de Engenharia Química \\ ${ }^{3}$ Universidade Regional de Blumenal, Departamento de Engenharia Química \\ ${ }^{4}$ Universidade Tecnológica Federal do Paraná, Departamento de Matemática \\ E-mail para contato: regiani_al@hotmail.com
}

\begin{abstract}
RESUMO - A técnica SAS (Supercritical Antissolvent) permite a cristalização de uma infinidade de drogas e biopolímeros sem que estes sofram danos ou degradação físicoquímicas. A obtenção de partículas com morfologia e tamanho controlados está relacionada a determinação de uma combinação de parâmetros de operação adequada a cada sistema de solvente e soluto. Neste trabalho um estudo numérico a respeito do impacto da variação da temperatura e pressão de operação no escoamento da câmara SAS é apresentado, pois próximo ao ponto crítico da mistura o raio das partículas formadas exibem alta sensibilidade a estas variáveis. Observaram-se variações de temperatura em torno de $5 \mathrm{~K}$; os campos de pressão se mantiveram praticamente constantes para as condições analisadas. A equação de estado de Peng-Robinson foi empregada para prever a sensibilidade da densidade da mistura à variações incrementais de temperatura e pressão indicando que uma formulação incompressível e não isotérmica do modelo é viável.
\end{abstract}

\section{INTRODUÇÃO}

Técnicas para precipitação de micro e nanopartículas empregando fluido em estado supercrítico têm sido modificadas e exploradas para aplicações nas mais diversas áreas incluindo a indústria farmacêutica, cosmética, alimentícia e outras como alternativas aos processos tradicionais (Balcão, et al., 2013; E.M. Berends, 1993; Martín, et al., 2007).

A técnica SAS (Supercritical Antisolvent) que emprega $\mathrm{CO}_{2}$ como antissolvente para a precipitação de partículas em escala micro e nanométrica permite o processamento de uma grande variedade de produtos de alta qualidade (Jerzy, et al., 2004). E requer que o solvente orgânico possua uma afinidade maior pelo antissolvente do que pelo soluto. Desta maneira, quando a solução contendo o soluto é posta em contato com o antissolvente em condições supercríticas, a solubilidade do soluto no solvente orgânico é diminuída levando à precipitação do soluto na forma particulada (Jung e Perrut, 2001). Na técnica SAS, próximo ao ponto crítico 


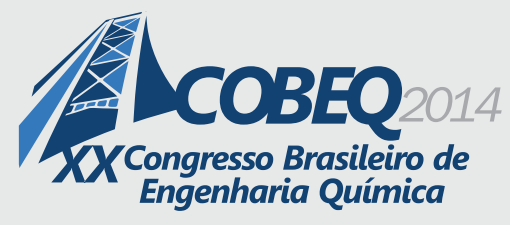

19 a 22 de outubro de 2014
Florianópolis/SC

da mistura as gotículas formadas exibem alta sensibilidade a variações de temperatura e de pressão. Estas duas variáveis apresentam grande influência nas mudanças de morfologia das substâncias precipitadas (De Marco e Reverchon, 2011) e comportamentos contraditórios em relação a variação dos tamanhos de partículas com estas variáveis podem ser apontados na literatura. Um aumento na pressão de operação resulta em diminuição no tamanho de partículas (Franceschi, 2009; Lengsfeld, et al., 2000). Quanto ao aumento na temperatura alguns solutos diminuem de tamanho enquanto outros aumentam, estas discrepâncias de resultados dependem da natureza física do soluto e ainda requerem mais estudos (Erriguible, et al., 2013). A modulação da pressão e da temperatura influi diretamente na variação da densidade e por sua vez, a dimensão dos tamanhos das partículas precipitadas depende da diferença de densidade entre regiões ricas em solvente orgânico e regiões ricas em $\mathrm{CO}_{2}$ (Werling e Debenedetti, 2000).

Neste trabalho é apresentada a partir do software ANSYS FLUENT uma análise do escoamento da mistura binária $\mathrm{CO}_{2}$ e Etanol no processo SAS. Tal análise enfatiza a variação que a pressão e a temperatura sofrem na câmara quando o processo é operado na região do diagrama de fases acima do ponto crítico da mistura (região de uma única fase). É feito também um estudo de quanto a densidade da mistura é sensível a variações incrementais de pressão e de temperatura em torno de seus valores de operação.

\section{MODELO MATEMÁTICO E CONDIÇÕES DE OPERAÇÃO}

Foram simulados dez casos: sob as temperaturas de operação $308 \mathrm{~K}, 313 \mathrm{~K}, 315 \mathrm{~K}, 317 \mathrm{~K}$ e $320 \mathrm{~K}$ à pressão de operação de 80 bar; e depois à pressão de operação de 120 bar. A densidade da mistura $\mathrm{CO}_{2}$ e Etanol foi descrita pela equação de estado de Peng-Robinson (PREOS):

$$
P=\frac{R T}{v-b_{m}}-\frac{a_{m}}{v\left(v+b_{m}\right)+b_{m}\left(v-b_{m}\right)}
$$

onde $P$ é a pressão absoluta, $v$ o volume molar, $T$ a temperatura, $a_{m}$ e $b_{m}$, são parâmetros da PREOS calculados empregando a regra de mistura quadrática de van der Waals com parâmetros de interação binários $k_{12}=0,0703$ e $l_{12}=-0,0262$ calculados a partir de dados de equilíbrio de fases por Franceschi (2009) para as presentes condições.

\subsection{Equações de conservação}

Foi considerado o escoamento em estado estacioário, não isotérmico, compressível de fluido Newtoniano e turbulento. A modelagem da turbulência será descrita via equações médias de Reynolds (RANS-Reynolds Averaged Navier Stokes). A decomposição de Reynolds descreve os valores instantâneos das variáveis do movimento turbulento como uma variação randômica em torno de seus valores médios (aqui será empregada a média ponderada pela densidade $\phi(x) \equiv \overline{\rho \phi} / \bar{\rho}$ onde a barra denota a média de Reynolds) (Wilcox, 1993) a partir do modelo de turbulência $k-\varepsilon$. Resultando nas equações médias de conservação da massa, da quantidade de 
movimento, da energia e a equação de transporte para a fração de mistura e para a variância da fração de mistura respectivamente:

$\partial_{i}\left(\bar{\rho} \tilde{u}_{i}\right)=0$

$\partial_{\mathrm{i}}\left(\bar{\rho} \tilde{u}_{i} \tilde{u}_{j}\right)=\partial_{j} \bar{P}+\partial_{j}\left(\tilde{\tau}_{i j}-\bar{\rho} u_{i}^{\prime} u_{j}^{\prime}\right)$

$\partial_{i}\left(\bar{\rho} \tilde{u}_{i} \tilde{h}\right)=\partial_{j}\left\{\left[\left(K_{m}+K_{T}\right) / c_{p}\right] \partial_{j} \tilde{h}\right\}+\tilde{\tau}_{i j} \partial_{j} u_{i}+\bar{\rho} u_{i}\left(\frac{\partial Q_{m}}{\partial y_{C o_{2}}} y_{1}+Q_{m}\right) \frac{\partial y_{1}}{\partial y_{C o_{2}}}$

$\partial_{j}\left(\bar{\rho} \tilde{u}_{j} \tilde{f}\right)=\partial_{j}\left[\bar{\rho}\left(D_{m}+D_{T}\right) \partial_{j} \tilde{f}\right]$

$\partial_{j}\left(\bar{\rho} \tilde{u}_{j} \tilde{\sigma}^{2}\right)=\partial_{j}\left[\bar{\rho}\left(D_{m}+D_{T}\right) \partial_{j} \tilde{\sigma}^{2}\right]+2 D_{T}\left(\partial_{j} \tilde{f}\right)^{2}-2 \frac{\varepsilon}{k} \tilde{\sigma}^{2}$

na Eq. (2) $-\bar{\rho} u_{i}^{\prime} u_{j}{ }_{j}$ é o tensor tensão de Reynolds, resolvido pelo modelo de turbulência (Tennekes, 1972), e $\tilde{\tau}_{i j}$ o tensor tensão viscoso, onde $u_{i}, i \in\{1,2,3\}$ são as componentes médias do vetor velocidade; na Eq. (3) $h$ é a entalpia total, $K_{m}$ e $K_{T}$ são a condutividade térmica molecular e condutividade térmica turbulenta respectivamente, $\mathrm{c}_{p} \mathrm{o}$ calor específico da mistura e o último termo do lado direito é o termo fonte devido ao calor de mistura $Q_{m}$ e a sua dependência da fração mássica de $\mathrm{CO}_{2} y_{\mathrm{Co} 2}$, é calculada pela equação de Peng-Robinson. Nas Eq. (5)-(6), $S c_{T}$ é o número de Schmidt turbulento, $D_{m}$ é a difusividade molecular e $D_{T}$ a difusividade turbulenta. A fração de mistura é a fração mássica de fluido alimentado no sistema a partir da entrada de solução $f=\left(y_{C O 2}-y_{\alpha, C o 2}\right) /\left(y_{\alpha, s o l}-y_{\alpha, C o 2}\right)$ (Fox, 2003). Onde $y_{\alpha, c o 2}$ é a fração mássica de $\mathrm{CO}_{2}$ na entrada de $\mathrm{CO}_{2}$ e $y_{\alpha, \text { sol }}$ a fração mássica de $\mathrm{CO}_{2}$ na entrada de solução respectivamente. E a variância da fração de mistura $\sigma^{2}$ representa o desvio da idealidade e o processo de mistura (Henczka e Shekunov, 2005).

\subsection{Propriedades Físicas da Mistura}

A viscosidade para cada componente foi calculada pela regra de Chung (Chung, et al., 1988) e a viscosidade da mistura foi calculada pela regra de mistura: $\mu_{m}=\exp \left(y_{\mathrm{Co}_{2}} \ln \left(\mu_{E \operatorname{tanol}}\right)+y_{E \operatorname{tanol}} \ln \left(\mu_{\mathrm{CO}_{2}}\right)\right)$. A condutividade térmica da mistura supercrítica também foi calculada a partir da regra de Chung (Poling, et al., 2004). E o coeficiente de difusão da mistura foi calculado pela da equação de Riazi and Whitson que leva em consideração a viscosidade e a densidade da mistura à baixas e à altas pressões (Riazi e Whitson, 1993). As propriedades físicas bem como as equações (5) e (6) foram implementadas em linguagem C. 


\subsection{Metodologia Numérica}

A câmara SAS considerada consiste de um tanque cilindrico que possui $120 \mathrm{~mm}$ de comprimento e $80 \mathrm{~mm}$ de diâmetro como considerado em Almeida, et al. (2012), acoplada à um capilar coaxial ao centro da tampa onde a entrada central de solução tem $0,01 \mathrm{~mm}$ de diâmetro e a entrada de $\mathrm{CO}_{2}$ tem $0,1 \mathrm{~mm}$ de diâmetro conforme detalhes na Figura 1.

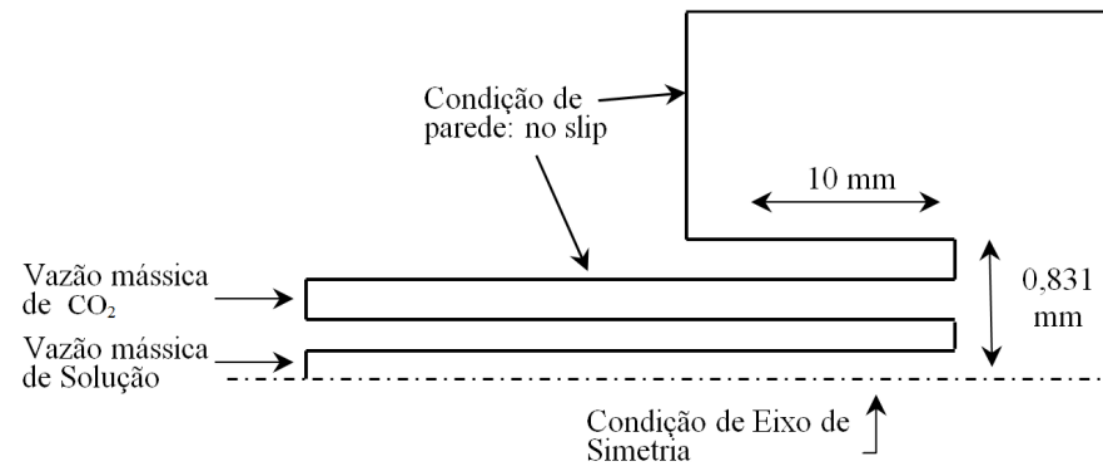

Figura 1- Detalhe da geometria da região do capilar coaxial da câmara SAS e condições de contorno para as Equações (2)-(6).

Após um estudo sobre a dependência da solução com a resolução da malha, foi empregada uma malha com $115,5 \times 10^{3}$ elementos mais refinada na região central do jato. As condições de contorno de saída foi de pressão manométrica nula. O solver segregado foi empregado através do algorítimo SIMPLE para resolver o acoplamento pressão-velocidade e o software FLUENT 13.0 foi empregado para resolver o sistema de equações linearizado.

\section{RESULTADOS E DISCUSSÃO}

\subsection{Diferencial de Densidade}

Considerando a temperatura e a pressão de operação de $T_{0}=313 \mathrm{~K}$ e $P_{0}=120 \mathrm{bar}$ respectivamente, usando o software Maple 15 a equação de estado cúbica de Peng-Robinson, Eq. (1), foi usada para prever o quanto a densidade da mistura $\rho$ é sensível a variações diferenciais de pressão e de temperatura em torno de seus valores de operação. Sabe-se que $d \rho=(\partial \rho / \partial P)_{T} d P+(\partial \rho / \partial T)_{P} d T$ e uma expansão em série de Taylor para $\rho$ truncada a partir do termo das suas segundas derivadas e para uma composição fixa $y_{i}^{0}$, resulta: $\rho\left(P+d P, T+d T, y_{1}^{0}\right)=\rho\left(P, T, y_{1}^{0}\right)+d P(\partial \rho / \partial P)_{T}+d T(\partial \rho / \partial T)_{P}$, e então pode-se quantificar como pequenas variações de $T$ e $P$ na câmara de precipitação influem na densidade da mistura pela equação: $\rho\left(P+d P, T+d T, y_{1}^{0}\right) \cong \rho\left(P, T, y_{1}^{0}\right)+d \rho$. 

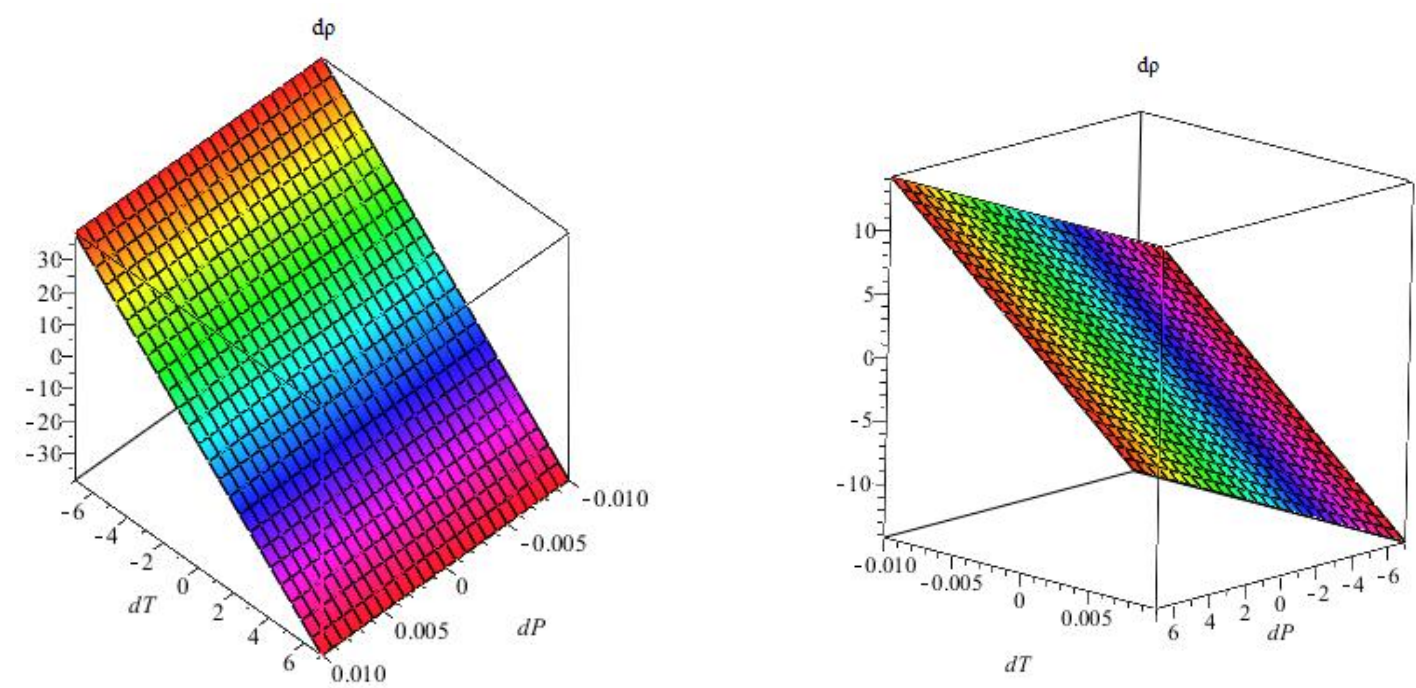

Figura 2 - Diferencial de densidade com $y_{l}=0.05$. (a) Com dT $= \pm 7 \mathrm{~K}$ e $\mathrm{dP}= \pm 0.01$ bar.

(b) Com $\mathrm{dP}= \pm 7$ bar com $\mathrm{dT}= \pm 0.01 \mathrm{~K}$.

Fixando $y_{1}=0,05$, observa-se que a variação de $d \rho$ com incrementos diferenciais de temperatura é maior (em módulo $|38.45| \mathrm{K}$ para $\mathrm{dP}=|0.01|$ bar) do que com incrementos diferenciais de pressão (em módulo $|14.181|$ bar para $\mathrm{dT}=|0.01| \mathrm{K}$ ) como na Figura 2. Este comportamento se mantém para composições $y_{1}<0,4$ e condições nas faixas de temperatura de operação $T_{0}$ de $280 \mathrm{~K}-325 \mathrm{~K}$ e de pressão de operação $P_{0}$ de 70 bar - 220 bar.

Assim pela última equação do parágrafo anterior a densidade da mistura variará em torno de 2,5 vezes a mais com a temperatura do que com a pressão. Observando que $7 \mathrm{~K}$ representa $2,2 \%$ da temperatura de operação $\left(T_{0}=313 \mathrm{~K}\right)$ e que 7 bar representa $5,8 \%$ da pressão de operação $\left(P_{0}=120\right.$ bar $)$. Como a pressão praticamente não varia no interior da câmara como mostra a Figura 4, as variações de densidade após a saída do capilar onde ocorre a nucleação das partículas (Lengsfeld, et al., 2000; Reverchon e De Marco, 2011) na câmara SAS são, nestas condições, devidas à temperatura e à composição da mistura.

\subsection{Variação de Temperatura}

A partir da adição do termo fonte calor de mistura na Eq. (4), foram obtidas variações de temperatura de $4 \mathrm{~K}$ para a pressão de operação $P_{0}=80$ bar e de $2 \mathrm{~K}$ para a pressão $P_{0}=120$ bar conforme a Figuras 3 logo após a saída do capilar. O que não ocorreu quando não se considerou tal termo e a variação das temperaturas ficaram em $1 \mathrm{~K}$ para as duas condições de pressão de operação. 


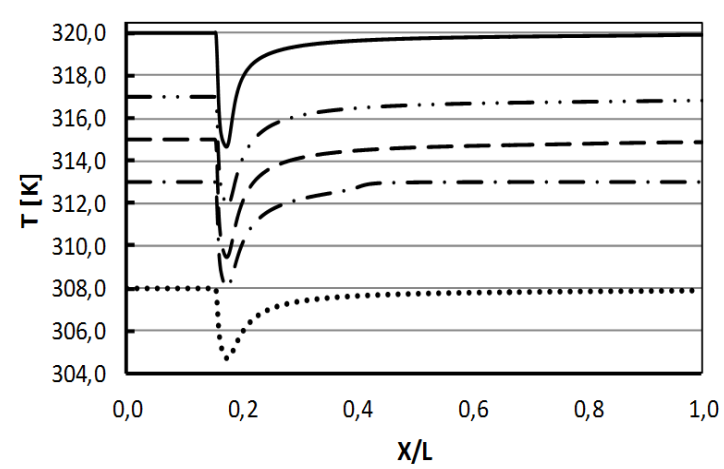

$P_{0}=80 \mathrm{bar}$

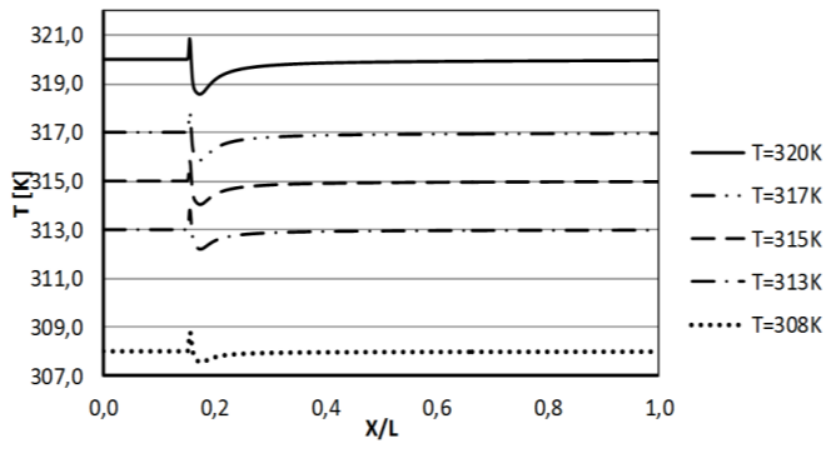

$P_{0}=120 \mathrm{bar}$

Figura 3-Variação de temperatura pela linha central axial da câmara adimensionalizada em $P_{0}=80 \mathrm{bar}$ (final do capilar $X / L \sim 0,15$ ).

\subsection{Variação de Pressão}

A pressão variou aproximadamente 0,9 bar no interior da câmara em torno de seu valor de operação para os dois casos considerados $\left(P_{0}=80\right.$ bar e $P_{0}=120$ bar $)$. Porém tais variações ocorreram no capilar de injeção, sendo que no interior da câmara para ambas as condições a pressões se mantiveram constantes como mostra a Figura 4.

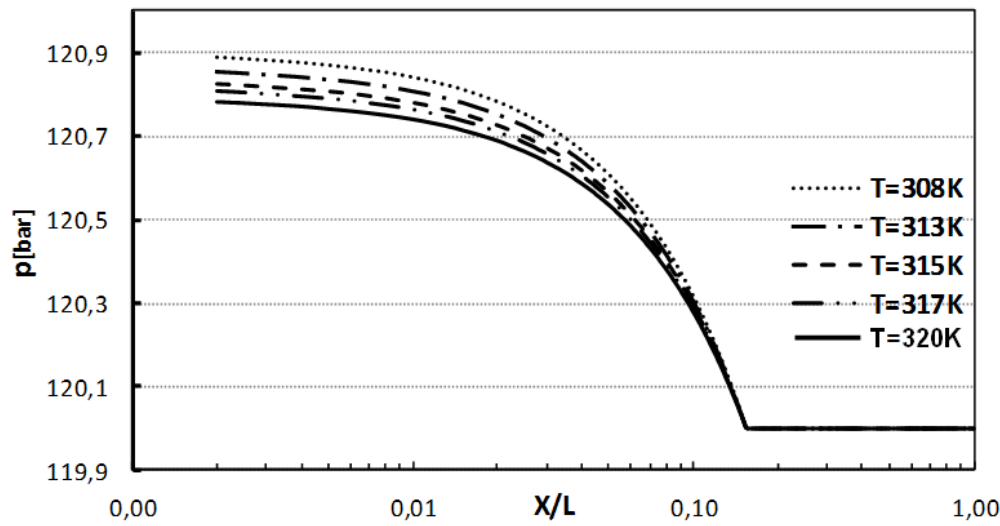

Figura 4 - Variação de pressão no interior da câmara para as temperaturas consideradas $X / L \sim 0,15$ corresponde a posição final do capilar.

\subsection{Eficiência da Mistura e Dependência das Propriedades Físicas}

A intensidade de segregação, $I_{s}=\tilde{\sigma}^{2} / \tilde{f}(1-\tilde{f})$ é igual a unidade quando os elementos do fluido não estão bem misturados na escala molecular e é igual a zero quando a mistura na escala molecular é considerada perfeita (Henczka e Shekunov, 2005). Para todos os casos $I_{s} \rightarrow 0$, para as temperaturas de operação consideradas e sob a condição de operação de $P_{0}=80$ bar os valores de $I s$ são mais altos em uma área maior do interior da câmara do que aqueles observados para a 
pressão $\mathrm{P}_{0}=120$ bar conforme Figura 5. Isto indica que há mistura mais eficiente na escala molecular à maior pressão de operação corroborando a observação experimental da literatura de que sob maior pressão são observadas menores tamanhos de partículas (De Marco e Reverchon, 2011; Franceschi, 2009).
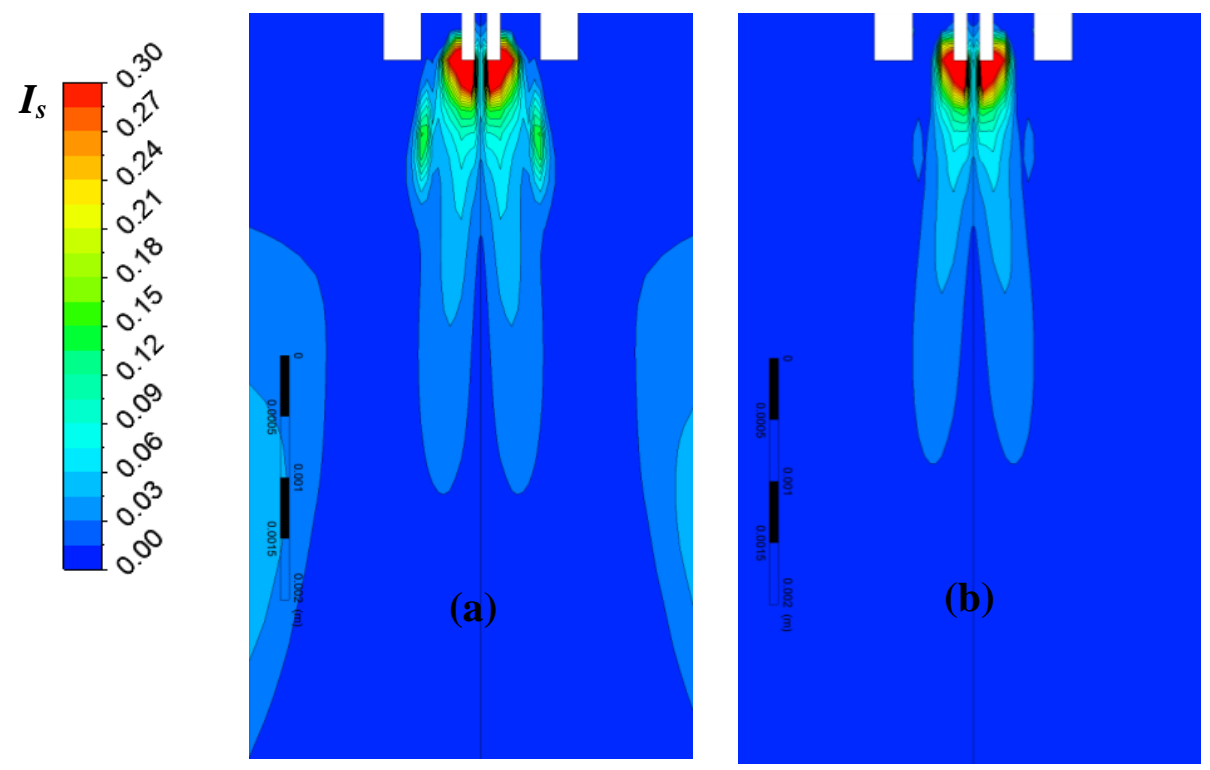

Figura 5 - Intensidade de segregação. (a) $\mathrm{P}_{0}=80$ bar; (b) $\mathrm{P}_{0}=120$ bar.

Quanto à importância das propriedades físicas da mistura dependentes de $T, P$ e $y_{i}$, ao considerá-las observou-se maior velocidade e menor quantidade de $\mathrm{CO}_{2}$ no interior da câmara em relação aos casos onde tais propriedades foram consideradas constantes, sendo estas características bastante pronunciadas somente para a pressão de operação de 80bar. Observou-se ainda que a viscosidade molecular, condutividade térmica e coeficiente de difusividade apresentaram variação expressiva somente na região da saída do capilar de injeção (até 4mm a frente) mantendo-se praticamente constantes no restante do interior da câmara de precipitação.

\section{CONCLUSÕES}

Um modelo matemático com as propriedades físicas: viscosidade molecular, condutividade térmica e coeficiente de difusividade dependentes da temperatura, pressão e composição da mistura foi proposto. Sob as condições consideradas, comparações com casos nos quais tais propriedades físicas foram consideradas constantes mostraram a necessidade da implementação desta dependência no modelo apenas para a menor pressão de operação.

A avaliação da influencia das variações diferenciais de temperatura e de pressão na densidade da mistura na câmara SAS mostrou que variações de pressão influem muito menos na densidade da mistura do que as variações de temperatura. Por sua vez, as variações de pressão na câmara de precipitação são pequenas e restritas ao capilar de injeção, assim para as condições testadas a consideração de uma formulação para a densidade dependente somente da temperatura 
e da composição da mistura seria uma boa estratégia numérica para a modelagem do escoamento na câmara SAS resultando em menos tempo e esforço computacional.

\section{REFERÊNCIAS}

ALMEIDA, R. A., et al. (2012). Modelagem Fluidodinâmca e Solução Numérica do Escoamento de Fluido Supercrítico Aplicados à Produção de Nanopartículas. Paper presented at the XIX Congresso Brasileiro de Engenharia Química.

BALCÃO, V. M., et al., Nanoencapsulation of bovine lactoferrin for food and biopharmaceutical applications. Food Hydrocolloids, v. 32. (2), p. 425-431, 2013.

CHUNG, T. H., et al., Generalized multiparameter correlation for nonpolar and polar fluid transport properties. Industrial \& Engineering Chemistry Research, v. 27. (4), p. 671-679, 1988.

DE MARCO, I., \& REVERCHON, E., Influence of pressure, temperature and concentration on the mechanisms of particle precipitation in supercritical antisolvent micronization. The Journal of Supercritical Fluids, v. 58. (2), p. 295-302, 2011.

E.M. BERENDS, O. S. L. B., G.M. VAN ROSMALEN, Nucleation and growth of fine crystals from supercritical carbon dioxide. Journal of Crystal Growth, v. Volume 128. p. 50-56, 1993.

ERRIGUIBLE, A., et al., Effect of pressure and non-isothermal injection on re-crystallization by $\mathrm{CO} 2$ antisolvent: Solubility measurements, simulation of mixing and experiments. The Journal of Supercritical Fluids, v. 76. (0), p. 115-125, 2013.

FOX, R. O. Computational Models for Turbulent Reacting Flows. United States of America: Cambridge University Press, New York, 2003.

FRANCESCHI, E. Precipitação e Encapsulamento de beta-Caroteno em PHBV Empregando Tecnologia Supercrítica. (Grau de Doutor). Engenharia de Alimentos., Universidade Federal de Santa Catarina, Florianópolis-SC., 2009

HENCZKA, M. B., JERZY., \& SHEKUNOV, B. Y., Particle formation by turbulent mixing with supercritical antisolvent. Chemical Engineering Science, v. 60. p. 2193- 2201, 2005.

JERZY, et al. Fluid Dynamics, Mass Transfer, and Particle Formation in Supercritical Fluids Supercritical Fluid Technology for Drug Product Development: Informa Healthcare, 2004.

JUNG, J., \& PERRUT, M., Particle design using supercritical fluids: Literature and patent survey. The Journal of Supercritical Fluids, v. 20. (3), p. 179-219, 2001.

LENGSFELD, C. S., et al., Mechanism Governing Microparticle Morphology during Precipitation by a Compressed Antisolvent: Atomization vs Nucleation and Growth. The Journal of Physical Chemistry B, v. 104. (12), p. 2725-2735, 2000.

MARTÍN, A., et al., Co-precipitation of carotenoids and bio-polymers with the supercritical anti-solvent process. The Journal of Supercritical Fluids, v. 41. (1), p. 138-147, 2007.

POLING, B. E., et al. The Properties of Gases and Liquids: McGraw-Hill Companies, 2004.

REVERCHON, E., \& DE MARCO, I., Mechanisms controlling supercritical antisolvent precipitate morphology. Chemical Engineering Journal, v. 169. (1-3), p. 358-370, 2011.

RIAZI, M. R., \& WHITSON, C. H., Estimating Diffusion Coefficients of Dense Fluids. Ind. Eng. Chem. Res., v., p. 3081-3088, 1993.

TENNEKES, H. J. L., LUMLEY. A First Course In Turbulence. London, England The Massachusetts Institute of Technology, 1972.

WERLING, J. O., \& DEBENEDETTI, P. G., Numerical modeling of mass transfer in the supercritical antisolvent process: miscible conditions. The Journal of Supercritical Fluids, v. 18. (1), p. 11-24, 2000.

WILCOX, D. C. Turbulence Modeling for CFD La Canada, California DCW Industries, 1993. 